Wilfrid Laurier University

Scholars Commons @ Laurier

Physics and Computer Science Faculty

Publications

Physics and Computer Science

2007

\title{
Quantum Ion-Acoustic Waves in Single-Walled Carbon Nanotubes Studied with a Quantum Hydrodynamic Model
}

Li Wei

Wilfrid Laurier University, Iwei@wlu.ca

You-Nian Wang

Dalian University of Technology

Follow this and additional works at: https://scholars.wlu.ca/phys_faculty

\section{Recommended Citation}

Wei, Li and Wang, You-Nian, "Quantum Ion-Acoustic Waves in Single-Walled Carbon Nanotubes Studied with a Quantum Hydrodynamic Model" (2007). Physics and Computer Science Faculty Publications. 82. https://scholars.wlu.ca/phys_faculty/82

This Article is brought to you for free and open access by the Physics and Computer Science at Scholars Commons @ Laurier. It has been accepted for inclusion in Physics and Computer Science Faculty Publications by an authorized administrator of Scholars Commons @ Laurier. For more information, please contact scholarscommons@wlu.ca. 


\title{
Quantum ion-acoustic waves in single-walled carbon nanotubes studied with a quantum hydrodynamic model
}

\author{
Li Wei* \\ Department of Physics and Computer Science, Wilfrid Laurier University, Waterloo, Ontario N2L 3C5, Canada \\ You-Nian Wang \\ Department of Physics, Dalian University of Technology, Dalian 116023, People's Republic of China \\ (Received 19 December 2006; revised manuscript received 3 March 2007; published 18 May 2007)
}

\begin{abstract}
The quantum ion-acoustic waves in single-wall carbon nanotubes are studied with the quantum hydrodynamic model, in which the electron and ion components of the nanotubes are regarded as a two-species quantum plasma system. An analytical expression of the dispersion relation is obtained for the linear disturbance. Numerical results show that the frequency of the ion-acoustic wave strongly depends on the nanotube's radius in the long-wavelength cases.
\end{abstract}

DOI: 10.1103/PhysRevB.75.193407

PACS number(s): 73.22.Lp, 52.35.Fp, 71.10.Ca

One of the most fascinating aspects about carbon nanotubes is their collective electronic excitations which may be very important in understanding the electron interactions in carbon nanotubes as well as the characteristics of their electronic structures. During the past years, the collective excitations have been widely studied with different theoretical models, such as the classical hydrodynamic model ${ }^{1,2}$ and the quantum dielectric-response model with the random-phase approximation. ${ }^{3-5}$ The studies correspond to high-frequency excitations which come from electron oscillations in carbon nanotubes.

It is well known that a carbon nanotube can be metallic or semiconducting, which depends on its radius and the geometric angle, and their electron and ion components can be regarded as a two-species quantum plasma system. Both the electrons and ions in the carbon nanotubes oscillate under the low-frequency disturbances. In contrast to the highfrequency excitations, we may expect that the low-frequency disturbances will lead to a new excitation in the carbon nanotube, i.e., quantum ion-acoustic wave mode. A powerful theoretical tool for studying the quantum plasma is the quantum hydrodynamic (QHD) model which was developed by Haas et al. ${ }^{6}$ The QHD has been recently used to the quantum dust acoustic wave $^{7}$ and instabilities of electromagnetic waves in quantum plasma. ${ }^{8}$ In particular, Haas studied ion-acoustic waves $^{9}$ in the one-dimensional quantum plasmas.

The aim of this paper is to study the dispersion relation of the quantum ion-acoustic wave in the carbon nanotube with the QHD model, in which we model a single-wall carbon nanotube as an infinitesimally thin and infinitely long cylindrical shell with a radius $a$, and consider that electrons and ions are distributed uniformly over the cylindrical surface, with the equilibrium density $n_{0}$. In the cylindrical coordinates $\mathbf{r}_{s}=(a, \phi, z)$, the electron (ion) density $n_{e}\left(n_{i}\right)$ and fluid velocity $\mathbf{u}_{e}\left(\mathbf{u}_{i}\right)$ can be determined by the following QHD equations: 6,9

$$
\begin{gathered}
\frac{\partial n_{e}}{\partial t}+\nabla_{\|} \cdot\left(n_{e} \mathbf{u}_{e}\right)=0, \\
\frac{\partial n_{i}}{\partial t}+\nabla_{\|} \cdot\left(n_{i} \mathbf{u}_{i}\right)=0,
\end{gathered}
$$

$$
\begin{gathered}
m_{e}\left[\frac{\partial \mathbf{u}_{e}}{\partial t}+\left(\mathbf{u}_{e} \cdot \nabla_{\|}\right) \mathbf{u}_{e}\right]=e \nabla_{\|} \Phi-\nabla_{\|} W_{e}+\frac{\hbar^{2}}{2 m_{e}} \nabla_{\|}\left(\frac{1}{\sqrt{n_{e}}} \nabla_{\|}^{2} \sqrt{n_{e}}\right) \\
m_{i}\left[\frac{\partial \mathbf{u}_{i}}{\partial t}+\left(\mathbf{u}_{i} \cdot \nabla_{\|}\right) \mathbf{u}_{i}\right]=-e \nabla_{\|} \Phi
\end{gathered}
$$

where $m_{e}\left(m_{i}\right)$ are the electron (ion) mass, $e$ is the charge, and $\hbar$ is the scaled Planck's constant; $\nabla_{\|}=\hat{\mathbf{e}}_{z} \frac{\partial}{\partial z}+\hat{\mathbf{e}}_{\phi} a^{-1} \frac{\partial}{\partial \phi}$, and $\Phi\left(\mathbf{r}_{s}, t\right)$ is the self-consistent potential. The second term on the right-hand side (RHS) of Eq. (3) is the force due to the internal interaction in the electron species, with $W_{e}=\frac{\pi \hbar^{2}}{m_{e}} n_{e}$ being the Fermi energy of the two-dimensional (2D) electron gas, and the last term on the RHS of Eq. (3) represents the quantum pressure which comes from the quantum diffraction effects.

In Eqs. (2) and (4), the self-consistent potential (in the CGS units) is given by

$$
\Phi\left(\mathbf{r}_{s}, t\right)=e \int d \mathbf{s}^{\prime} \frac{n_{i}\left(\mathbf{r}_{s}^{\prime}, t\right)-n_{e}\left(\mathbf{r}_{s}^{\prime}, t\right)}{\left|\mathbf{r}_{\mathbf{s}}-\mathbf{r}_{s}^{\prime}\right|}
$$

where $d \mathbf{s}^{\prime}=a d \phi^{\prime} d z^{\prime}$. By using the expansion of Coulomb potential, $1 /\left|\mathbf{r}_{s}-\mathbf{r}_{s}^{\prime}\right|$, in the cylindrical coordinates, the potential can be expressed by ${ }^{10}$

$$
\begin{aligned}
\Phi\left(\mathbf{r}_{s}, t\right)= & e \sum_{m=-\infty}^{\infty} \int_{-\infty}^{\infty} \frac{d k}{(2 \pi)^{2}} g(a, k, m) \int d \mathbf{s}^{\prime}\left[n_{i}\left(\mathbf{r}_{s}^{\prime}, t\right)\right. \\
& \left.-n_{e}\left(\mathbf{r}_{s}^{\prime}, t\right)\right] e^{i k\left(z-z^{\prime}\right)+i m\left(\phi-\phi^{\prime}\right)},
\end{aligned}
$$

where $g(a, k, m)=4 \pi I_{m}(k a) K_{m}(k a)$, while $I_{m}$ and $K_{m}$ are the cylindrical Bessel functions of order $m$.

Taking into account the small electron inertial force, i.e., $m_{e} / m_{i} \ll 1$, the left-hand side of Eq. (3) can be neglected under the low-frequency disturbance. Therefore, by integrating over Eq. (3) once and considering, the boundary conditions $n_{e}=n_{0}$ and $\Phi=0$ at $z=\infty$, we can obtain the relation between the electron density and the potential as follows: 


$$
e \Phi=W_{e}-W_{e 0}-\frac{\hbar^{2}}{2 m_{e} \sqrt{n_{e}}} \nabla_{\|}^{2} \sqrt{n_{e}},
$$

where $W_{e 0}=\frac{\pi \hbar^{2}}{m_{e}} n_{0}$.

The system of Eqs. (2), (4), (6), and (7) constitutes a set of nonlinear self-consistent equations for determining four unknown quantities, $n_{e}, n_{i}, \mathbf{u}_{i}$, and $\Phi$, which can only be solved numerically, in general. As usually done in the linear disturbance theory, we linearize the above equations by assuming $n_{e}=n_{0}+n_{e 1}, n_{i}=n_{0}+n_{i 1}, \mathbf{u}_{i}=\mathbf{u}_{i 1}$, and $\Phi=\Phi_{1}$, where $n_{e 1}, n_{i 1}, \mathbf{u}_{i 1}$, and $\Phi_{1}$ are the perturbed quantities. As a result, we obtain the following linearized equations:

$$
\begin{gathered}
\frac{\partial n_{i 1}}{\partial t}=-n_{0} \nabla_{\|} \cdot \mathbf{u}_{i 1}, \\
\frac{\partial \mathbf{u}_{i 1}}{\partial t}=-\frac{e}{m_{i}} \nabla_{\|} \Phi_{1}, \\
e \Phi_{1}=\frac{\pi \hbar^{2}}{m_{e}} n_{e 1}-\frac{\hbar^{2}}{4 m_{e} n_{0}} \nabla_{\|}^{2} n_{e 1}, \\
\Phi_{1}\left(\mathbf{r}_{s}, t\right)=e \sum_{m=-\infty}^{\infty} \int_{-\infty}^{\infty} \frac{d k}{(2 \pi)^{2}} g(a, k, m) \int d \mathbf{s}^{\prime}\left[n_{i 1}\left(\mathbf{r}_{s}^{\prime}, t\right)\right. \\
\left.-n_{e 1}\left(\mathbf{r}_{s}^{\prime}, t\right)\right] e^{i k\left(z-z^{\prime}\right)+i m\left(\phi-\phi^{\prime}\right)} .
\end{gathered}
$$

Here, $k$ is the lognitudinal wave number and $m$ is the discrete azimuthal quantum number.

We further apply the Fourier transform

$$
\begin{aligned}
A\left(\mathbf{r}_{s}, t\right)= & \sum_{m=-\infty}^{\infty} \int_{-\infty}^{\infty} \frac{d k}{(2 \pi)^{2}} \int_{-\infty}^{\infty} \frac{d \omega}{2 \pi} \\
& \times A(a, k, m, \omega) e^{i k\left(z-z^{\prime}\right)+i m\left(\phi-\phi^{\prime}\right)-i \omega t},
\end{aligned}
$$

where $A\left(\mathbf{r}_{s}, t\right)$ stands for any of the above-listed perturbed quantities. With this transform, one may express the Fourier transforms of the perturbed electron density, ion density, and potential as follows:

$$
\begin{aligned}
& n_{e 1}(a, k, m)=\frac{e m_{e}}{\pi \hbar^{2}} \frac{\Phi_{1}(a, k, m)}{1+k_{m}^{2} / 2 k_{F}^{2}}, \\
& n_{i 1}(a, k, m)=\frac{e n_{0} k_{m}^{2}}{m_{i} \omega^{2}} \Phi_{1}(a, k, m),
\end{aligned}
$$

and

$$
\Phi_{1}(a, k, m)=\operatorname{eag}(a, k, m)\left[n_{i 1}(a, k, m)-n_{e 1}(a, k, m)\right],
$$

where $k_{F}=\left(2 \pi n_{0}\right)^{1 / 2}$ is the Fermi wave number of the $2 \mathrm{D}$ electron gas and $k_{m}^{2}=k^{2}+m^{2} / a^{2}$. By substituting Eqs. (13) and (14) into Eq. (15), we can get the following dispersion relation for the linear quantum ion-acoustic wave:

$$
\omega^{2} / \omega_{s}^{2}=\left(k_{m} a_{B}\right)^{2} \frac{g(a, k, m)}{\pi a_{B} / a+2 k_{F}^{2} g(a, k, m) /\left(k_{m}^{2}+2 k_{F}^{2}\right)},
$$

where $\omega_{s}=c_{s} / a_{B}, a_{B}=\hbar^{2} /\left(e^{2} m_{e}\right)$ is the Bohr radius, $c_{s}$ $=\sqrt{T_{F} / m_{i}}$ is the ion-acoustic velocity, and $T_{F}=\frac{\pi \hbar^{2}}{m_{e}} n_{0}$ is the Fermi temperature of the 2D electron gas.

In the long-wavelength limit, i.e., $k a \rightarrow 0$, we can use the behavior of the $I_{m}(x)$ and $K_{m}(x)$ functions when $x \rightarrow 0$, namely, $\quad I_{m}(x) \rightarrow a_{m} x^{m}, \quad K_{0}(x) \rightarrow \ln (1.123 / x), \quad$ and $\quad K_{m}(x)$ $\rightarrow b_{m} x^{-m}(m \neq 0)$, where $\quad a_{m}=2^{-m} / \Gamma(m+1)$ and $b_{m}$ $=2^{m-1} \Gamma(m)$, then we can get, for $m=0$,

$$
\omega=0 \text {, }
$$

and for $m \neq 0$,

$$
\omega^{2} / \omega_{s}^{2}=\frac{2 m^{2}\left(a_{B} / a\right)^{2}}{m a_{B} / a+4 k_{F}^{2} /\left(2 k_{F}^{2}+m^{2} / a^{2}\right)},
$$

which depends strongly on the radius of the nanotube.

In contrast, we may use the asymptotic expressions of the Bessel functions $I_{m}(x)=e^{x} / \sqrt{2 \pi x}$ and $K_{m}(x)=\sqrt{\frac{\pi}{2 x}} e^{-x}$ when $x \rightarrow \infty$. Thus, the dispersion relation can be written approximately as

$$
\omega^{2} / \omega_{s}^{2}=\frac{\left(k_{m} a_{B}\right)^{2}}{k a_{B} / 2+2 k_{F}^{2} /\left(k_{m}^{2}+2 k_{F}^{2}\right)} .
$$

Especially, when the nanotube radius becomes infinity, i.e., $a \rightarrow \infty$, Eq. (19) can be reduced to

$$
\omega^{2} / \omega_{s}^{2}=\frac{\left(k a_{B}\right)^{2}}{k a_{B} / 2+2 k_{F}^{2} /\left(k^{2}+2 k_{F}^{2}\right)},
$$

which is the dispersion relation of the ion-acoustic wave in a 2D planar quantum plasma.

It can be seen from Eq. (16) that the dispersion relation depends on the tubule's radius $a$ and the surface electron density $n_{0}$. Generally, radii of the single-wall carbon nanotubes range from $1 \mathrm{~nm}$ up to almost $15 \mathrm{~nm}$. Using the atomic density of a graphite sheet $38 \mathrm{~nm}^{-2}$, the surface electron density of a single-walled carbon nanotube can be approximated by $n_{0}=4 \times 38 \mathrm{~nm}^{-2} .11$ To further reveal the characteristics of the dispersion relation in the long-wavelength limit, we plotted the dependence of the normalized frequency $\omega / \omega_{s}$ on the variable $k a_{B}$ for different nanotube's radii $a$ with fixed $m$ $=2$ in Fig. 1. One can see that for long-wavelength region (i.e., $k a_{B} \rightarrow 0$ ), as increasing the nanotube's radius the normalized frequency $\omega / \omega_{s}$ decreases, while the normalized frequency approaches to each other for short-wavelength region (i.e., $\left.k a_{B} \rightarrow \infty\right)$. To see the effect of the discrete azimuthal quantum number $m$ on the normalized frequency, we plotted the normalized frequency as a function of $k a_{B}$ for different values of $m$ with fixed $a=5 a_{B}$ in Fig. 2. It can be seen from Fig. 2 that for long-wavelength region, as increasing the number of $m$, the normalized frequency increases, and for short-wavelength region, the same asymptotic behavior as that in Fig. 1 is observed.

In summary, we have used the QHD model to describe the propagation of the quantum ion-acoustic wave in the singlewall carbon nanotues. An analytical expression of the disper- 


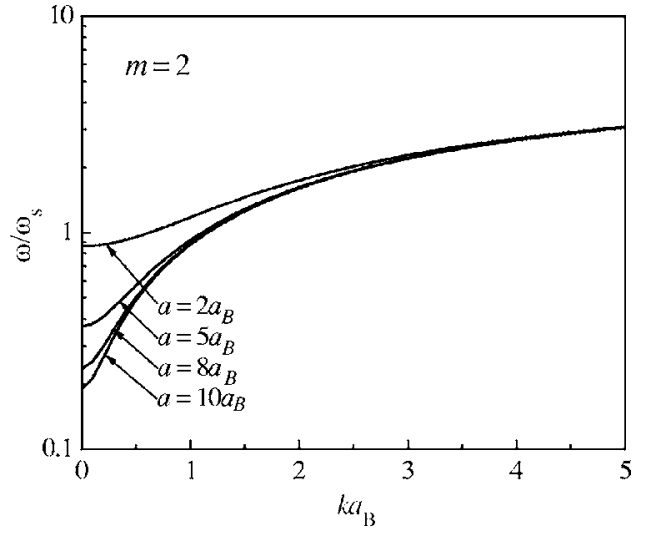

FIG. 1. The dispersion relation $\omega / \omega_{s}$ of the quantum ionacoustic wave for the discrete azimuthal quantum numbers $m=2$ and different nanotube's radii: $a=2 a_{B}, a=5 a_{B}, a=8 a_{B}$, and $a=10 a_{B}$.

sion relations has been derived in the case of the linear disturbance. Simulation results show that for a fixed surface electron density of a single-walled carbon nanotube, the frequencies of the ion-acoustic waves strongly depend on the

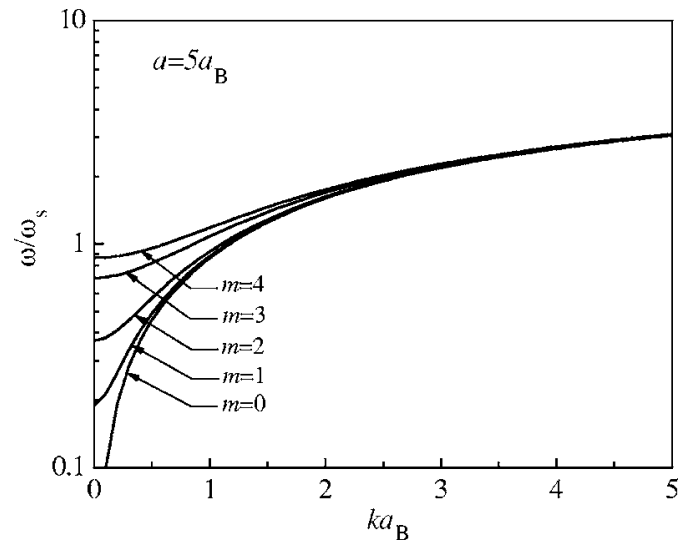

FIG. 2. The dispersion relation $\omega / \omega_{s}$ of the quantum ionacoustic wave for the nanotube's radius $a=5 a_{B}$ and different azimuthal quantum numbers $m=0,1,2,3$, and 4 .

nanotube's radius and the azimuthal quantum number in the long-wavelength cases.

This work was supported by the Natural Sciences and Engineering Research Council of Canada.
*Electronic address: lwei@wlu.ca

${ }^{1}$ C. Yannouleas, E. N. Bogachek, and U. Landman, Phys. Rev. B 53, 10225 (1996).

${ }^{2}$ X. Jiang, Phys. Rev. B 54, 13487 (1996).

${ }^{3}$ O. Sato, Y. Tanaka, M. Kobayashi, and A. Hasegawa, Phys. Rev. B 48, 1947, (1993).

${ }^{4}$ M. F. Lin and Kenneth W.-K. Shung, Phys. Rev. B 47, 6617 (1993).

${ }^{5}$ M. F. Lin, D. S. Chuu, C. S. Huang, Y. K. Lin, and K. W.-K. Shung, Phys. Rev. B 53, 15493 (1996).
${ }^{6}$ F. Haas, G. Manfredi, and M. Feix, Phys. Rev. E 62, 2763 (2000).

${ }^{7}$ P. K. Shukla and S. Ali, Phys. Plasmas 12, 114502 (2005).

${ }^{8}$ P. K. Shukla and L. S. Stenflo, Phys. Plasmas 13, 044505 (2006).

${ }^{9}$ F. Haas, L. G. Garcia, J. Goedert, and G. Manfredi, Phys. Plasmas 10, 3858 (2003).

${ }^{10}$ J. D. Jackson, Classical Electrodynamics (Wiley, New York, 1975).

${ }^{11}$ D. Ostling, D. Tomanek, and A. Rosen, Phys. Rev. B 55, 13980 (1997). 